\title{
Furthering the prostate cancer screening debate (prostate cancer specific mortality and associated risks)
}

\author{
G. Michael Allan MD, CCFP,; Michael P. Chetner, MD, MSc, FRCSC, FACS, ${ }^{\dagger}$ Bryan J. Donnelly, MCh, MSc,

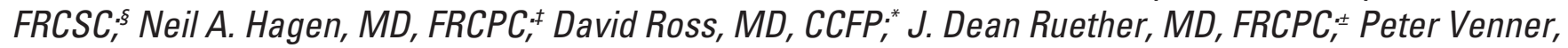 \\ $M D ;{ }^{* *}$ as part of the Toward Optimized Practice Prostate Cancer Working Group
}

\begin{abstract}
*Department of Family Medicine, University of Alberta, Edmonton, AB; 'Department of Surgery, Faculty of Medicine, University of Alberta, Edmonton, AB; \$Prostate Cancer Centre, Calgary, AB; ; of Oncology, Clinical Neurosciences and Medicine, University of Calgary, Calgary, AB; *Department of Medical Oncology, University of Calgary, Calgary, AB; ${ }^{*}$ Department of Medical Oncology, Cross Cancer Institute, \& Division of Medical Oncology, Department of Oncology, University of Alberta, Edmonton, AB
\end{abstract}

Cite as: Can Urol Assoc J 2011;5(6):416-21; http://dx.doi.org/10.5489/cuai.11063

\begin{abstract}
Screening for prostate cancer remains a contentious issue. As with other cancer screening programs, a key feature of the debate is verification of cancer-specific mortality reductions. Unfortunately the present evidence, two systematic reviews and six randomized controlled trials, have reported conflicting results. Furthermore, half of the studies are poor quality and the evidence is clouded by key weaknesses, including poor adherence to screening in the intervention arm or high rates of screening in the control arm. In high quality studies of prostate cancer screening (particularly prostatespecific antigen), in which actual compliance was anticipated in the study design, there is good evidence that prostate cancer mortality is reduced. The numbers needed to screen are at least as good as those of mammography for breast cancer and fecal occult blood testing for colo-rectal cancer. However, the risks associated with prostate cancer screening are considerable and must be weighed against the advantage of reduced cancer-specific mortality. Adverse events include $70 \%$ rate of false positives, important risks associated with prostate biopsy, and the serious consequences of prostate cancer treatment. The best evidence demonstrates prostate cancer screening will reduce prostate cancer mortality. It is time for the debate to move beyond this issue, and begin a well-informed discussion on the remaining complex issues associated with prostate cancer screening and appropriate management.
\end{abstract}

\section{Introduction}

Screening for prostate cancer remains contentious. Two primary methods for screening currently exist: the digital rectal exam (DRE) and the prostate-specific antigen (PSA) laboratory test. Uncertainties surround prostate cancer screening, including questions about reduction in overall mortality, prostate cancer specific mortality, and the cost/ benefit ratio associated with screening. False positives and false negatives, the potential harm from workup and treatment, and the subsequent effects on quality of life com- pound the complexity of this issue. As with other cancer screening programs, a key feature of the debate is verification of cancer-specific mortality reductions. In this paper, we will review the available evidence regarding the impact of prostate cancer screening on prostate cancer mortality. Although total mortality is clearly an important outcome, the adoptions of other large cancer screening programs (e.g., breast and colon cancer) were based on reductions in cancer-specific mortality. Additionally, we will consider some of the risks and harms associated with prostate cancer screening to promote an informed debate.

\section{Systematic review and meta-analysis}

Ideally, a systematic review summarizing similar studies of screening effects on prostate cancer mortality would provide clear direction to further the debate. In the last few years, two systematic reviews and meta-analyses have attempted to distill the effects of prostate cancer screening. ${ }^{1,2}$

In their 2010 review, Djulbegovic and colleagues identified six studies. ${ }^{1}$ The data revealed that screening increased the diagnosis of prostate cancer in general (risk ratio [RR] 1.46 , confidence interval $[\mathrm{Cl}] 1.21$ to $1.77 ; p<0.001)$ and specifically stage I prostate cancer (RR 1.95, CI 1.22 to 3.13; $p=0.005)$. Screening did not result in a statistically significant reduction in overall mortality (RR 0.99, Cl 0.97 to $1.01 ; p=0.44$ ) or prostate cancer-specific mortality (RR $0.88, \mathrm{Cl} 0.71$ to $1.09 ; p=0.25)$. The results of statistical testing of heterogeneity among prostate cancer mortality demonstrated important heterogeneity $\left(\mathrm{I}^{2}=55 \%, \chi^{2}=8.89\right.$; $p=0.06)$. Although heterogeneity testing itself must be interpreted with caution, ${ }^{3,4}$ this value suggests that important differences may exist.

In their 2011 review, Ilic and colleagues identified five studies. ${ }^{2}$ Data revealed that screening increased the diagnosis of prostate cancer in general (RR 1.35, Cl 1.06 to 1.72). Screening did not result in a statistically significant reduction in overall mortality (RR $1.00, \mathrm{Cl} 0.98$ to 1.02 ) or prostate 
cancer-specific mortality (RR $0.95, \mathrm{Cl} 0.85$ to 1.07 ). Of the five included studies, three had a high risk of bias due to methodological weaknesses. Of the remaining two, one was considered to have short follow-up and received minimal weighting in the meta-analysis.

We believe these meta-analyses obscure, not enhance, the issue of prostate cancer screening's effect on cancer-specific mortality. By combining studies that vary considerably and have different but important limitations, these metaanalyses mask potential information that can be gleaned from individual randomized controlled trials (RCTs).

\section{RCTs assessing prostate cancer screening on prostate cancer mortality}

Six RCTs of prostate cancer screening provide results of prostate cancer mortality. ${ }^{5-14}$ Seven different RCTs were included in the two systematic reviews, but one did not provide data on prostate cancer mortality and was not further discussed. ${ }^{15}$ We summarized remaining six studies (Table 1, Table 2). The type of screening, randomization characteristics, blinded outcome assessment and duration of follow-up are listed, along with their respective systematic reviews (Table 1 ). Traditional blinding of clinicians and patients would not be expected in screening studies and was not summarized. Table 2 includes the number of patients randomized to each arm, power calculations, percent of patients complying in each arm, and the screening effect on prostate cancer mortality in both the intention to screen and per protocol analyses. Data are abstracted from the original publications, ${ }^{5-14}$ but also compared with the two systematic reviews ${ }^{1,2}$ and ACP Journal Club summaries of articles. ${ }^{16-18}$

An examination of clinical and methodological characteristics among the individual RCTs will help in understanding the variations in trial design and outcomes that affect application. Herein, we discuss the specific concerns of each study.

\section{Norrkoping study}

The Norrkoping study ${ }^{5,6}$ involved all men between the ages of 50 and 69 in Norrkoping, Sweden. One in every six men was "randomly" selected from a list of birthdays (quasi-randomization) to be screened for prostate cancer, with the remainder serving as controls. There are important methodological issues with this study, including lack of clarity on allocation concealment and whether outcome assessment was blinded. In addition, there was no reporting of compliance (contamination) in the unscreened population. The authors did not describe power calculations, but the small numbers in the screening arm were underpowered to find a statistically significant difference in prostate cancer mortal- ity. PSA screening was not included until the study's sixth year, so the study had 10 (not 15) years of PSA follow-up. In our view, the Norrkoping study was sufficiently flawed that it should not influence the debate on prostate cancer screening.

\section{Quebec study}

In the Quebec study, ${ }^{7,8}$ all 45 to 80-year-old men in Quebec City were randomized on a 2:1 ratio to prostate cancer screening or no screening. This study had multiple methodological issues, including unclear randomization, allocation concealment, blinding of outcome assessment and power calculation.

The greatest concern with this study was the very poor compliance with the screening intervention. Screening occurred in only $23.6 \%$ of the group randomized to screening compared to $7.3 \%$ of the group randomized to no screening. In other words, $76.4 \%$ of the screening population never received the intended intervention. Screening occurred in only $16.3 \%$ more of the screened group than in the unscreened group. The authors stated that they randomized twice the number of men to screening to account for the anticipated poor compliance, but provided no description of power calculation or the anticipated rate of compliance. Based on the available formula to determine sample size accounting for non-compliance, ${ }^{19}$ the trial would have required over half a million men per arm to find a difference with the same power.

It is not surprising, therefore, that the intention-to-screen analysis found no statistical difference between the two study populations. A per-protocol analysis (comparing those actually screened versus those not screened) reveals a clear statistically significant reduction in prostate cancer mortality (relative risk $0.36, \mathrm{Cl} 0.19$ to 0.65 ). Per-protocol analysis is subject to considerable bias, but the difference between the two analyses (intention to screen and per protocol) shows that compliance to protocol may have profoundly influenced the outcome. Poor adherence in the Quebec study was so overwhelming that an approximation of prostate cancer screening's effect on prostate cancer mortality is impossible.

\section{PLCO}

The PLCO (Prostate, Lung, Colorectal and Ovarian) study9,10 was a large, well-designed trial enrolling 55 to 74 -year-old men from 10 American centres. Key methodological characteristics were adequate and well-described. The concerns with PLCO, though not as substantial as other trials, are still important and potentially limit its application.

About $44 \%$ of men had one or more PSA tests in the three years before the study, potentially reducing the probability of prostate cancer detection in these men during the 
Allan et al.

conduct of the trial. Vital status was available for $98 \%$ of the population at seven years of follow-up, but only $67 \%$ by 10 years. Given that this group was potentially at lower risk at study entrance (due to pre-screening), longer study duration would likely be needed to see changes in prostate cancer mortality. Lastly, the no-screening arm suffered from high non-compliance or "contamination." Some screening occurred in $86 \%$ of the screening group versus $52 \%$ of the no-screening group - a difference of 34\%. The authors planned for non-compliance in their power calculations, anticipating 90\% compliance in the screening and $20 \%$ non-compliance or contamination in the no-screening arm. ${ }^{10}$ To maintain the same level of power, PLCO would have needed to find 2 or more times the benefit anticipated to reach statistical significance. ${ }^{10}$ In light of these limitations, it is not surprising the study did not identify a statistically significant reduction in prostate cancer mortality. We considered the PLCO results, but its limitations prevent a clear understanding of the potential effect of PSA screening on prostate cancer specific mortality compared to no screening in a normal risk population.

A recently published sub-group analysis of PLCO has shown that prostate cancer screening reduced prostate cancer mortality in men with no or minimal comorbidity (adjusted hazard ratio [HR], 0.56, $\mathrm{Cl} 0.33$ to 0.95), but not in men with at least one significant comorbidity (adjusted HR 1.43, $\mathrm{Cl} 0.96$ to 2.11). ${ }^{20}$ Although this result supports prostate cancer screening in healthy older men, the subgroup analysis should be interpreted with caution.

\section{ERSPC}

The ERSPC (European Randomized Study of Screening for Prostate Cancer) study ${ }^{11}$ involved seven linked, but different, randomized trials in seven European countries. This is the largest study of prostate cancer screening to date and was generally well-designed, but had certain limitations. The protocol in this trial varied between countries in enrollment (some randomized from the population, others enrolled before randomization), DRE use (some included early, but generally stopped over time) and different intervals for PSA testing and cut-off values that triggered further investigations. It is reassuring that the prostate cancer mortality rate ratios for screened and unscreened populations did not vary considerably (0.74 to 0.84$)$ between study centres (countries). The ERSPC was the first true randomized trial in which there was at least a $50 \%$ difference in the rates of screening when comparing the screened to the noscreened population. The per-protocol analysis improved the rate ratio, but not to an important degree. ${ }^{12}$ This finding suggests that the lack of compliance with the study protocol was less problematic in ERSPC, and supports the intentionto-screen analysis. In our view, the ERSPC provided a valid approximation of prostate cancer specific mortality reductions from PSA screening compared to no screening in a normal risk population.

\section{Göteborg}

The Göteborg study ${ }^{13}$ of Sweden randomized 20000 men directly from the Göteborg population registry. The study had extended follow-up (14 years) and was generally welldesigned. Although included in the ERSPC, the Göteborg study was designed independently and started before ERSPC. Limitations included a change in protocol partway through the study (reducing the PSA cut-off from 3 to $2.5 \mathrm{ng} / \mathrm{mL}$ ) and a lack of clarity regarding contamination in the noscreening arm (reported as "low"). The Göteborg study found the largest reduction in prostate cancer mortality (rate ratio 0.56). This changed little in the per protocol analysis, supporting the findings of the intention-to-screen analysis. The benefit observed in the Göteborg trial may have resulted, in part, from a meaningful difference in screening between the two randomized groups, a low rate (3\%) of pre-trial PSA screening and long follow-up. In our view, the Göteborg trial provided a valid approximation of prostate cancer-specific mortality reductions from PSA screening compared to no screening in a normal risk population.

\section{Stockholm}

The Stockholm study had two parts: (1) tallying the overall and prostate cancer-specific mortality effect of a one-time screening for prostate cancer ${ }^{14}$ and (2) determining the influence of dihydrotestosterone levels on survival from prostate cancer. ${ }^{21}$ The former is relevant to this review. All men age 55 to 70 were selected from the catchment area of Stockholm South Hospital. Of these 26602 men, 2400 (9\%) were randomly selected for screening. Of those, 1782 (74\%, or $6.7 \%$ of the original population) participated in the one round of screening with DRE, PSA and transrectal ultrasound (TRUS). Positive findings on DRE and TRUS led to biopsy. For PSA greater than 7, the TRUS was repeated and prostate biopsy was done if an abnormality was found. Biopsy was performed for any PSA greater than 10 .

As detailed in Table 1 and Table 2, there are multiple methodological concerns with the Stockholm study. Important information around randomization, allocation concealment, blinded outcome assessment, power calculation and compliance (contamination) in the unscreened population are all unclear or not available. However, the biggest limitation of the Stockholm study is the inadequate screening. A screening program based on one round of screening and biopsies only for PSA results greater than 10 would not be considered reasonable. As the design is not reflective of true population screening programs, external 
The continuing debate on PCa screening

\begin{tabular}{|c|c|c|c|c|c|c|c|}
\hline & \multicolumn{2}{|c|}{ Systematic review } & \multirow[t]{2}{*}{ Screening methods } & \multirow[t]{2}{*}{ Randomization } & \multirow[t]{2}{*}{$\begin{array}{l}\text { Allocation } \\
\text { concealment }\end{array}$} & \multirow[t]{2}{*}{$\begin{array}{l}\text { Blinded outcome } \\
\text { assessment }\end{array}$} & \multirow[t]{2}{*}{$\begin{array}{c}\text { Total } \\
\text { follow-up }\end{array}$} \\
\hline & BMJ 2010 & BJU Int 2011 & & & & & \\
\hline $\begin{array}{l}\text { Norrkoping, } \\
\text { Sweden }\end{array}$ & Yes & Yes & $\begin{array}{l}\text { DRE every } 3 \text { years } \times 4 \\
\text { PSA every } 3 \text { years } \times 2^{\dagger}\end{array}$ & Quasi* & Unclear & Unclear & $\begin{array}{l}15 \text { years } \\
(10 \mathrm{PSA})^{\dagger}\end{array}$ \\
\hline Quebec City ${ }^{7,8}$ & Yes & Yes & $\begin{array}{l}\text { DRE + PSA } \\
\text { PSA yearly }\end{array}$ & Unclear & Unclear & Unclear & 11 years \\
\hline PLCO (USA) ${ }^{9,10}$ & Yes & Yes & $\begin{array}{l}\text { PSA every year } \times 6 \\
\text { DRE every year } \times 4\end{array}$ & Adequate & Adequate & Yes & 11.5 years \\
\hline $\begin{array}{l}\text { ERSPC } \\
\text { (Europe) })^{11,12}\end{array}$ & Yes & Yes & $\begin{array}{l}\text { PSA every } 4 \text { years } \\
\text { (early DRE) }\end{array}$ & Adequate & Unclear & Yes & 9 years \\
\hline $\begin{array}{l}\text { Goteborg } \\
\text { (Sweden) })^{13}\end{array}$ & Yes & No & PSA every 2 years & Adequate & Adequate & Yes & 14 years \\
\hline Stockholm ${ }^{14}$ & No & Yes & $\begin{array}{l}\text { DRE, PSA, TRUS all } \\
\text { once }\end{array}$ & Unclear & Unclear & Unclear & 12.9 years \\
\hline
\end{tabular}

application of this study is difficult and inclusion in a metaanalysis of PSA screening is inappropriate and misleading.

\section{Summarizing the quality of the six studies}

When considering the quality of the six studies, it is easy to differentiate the high from low quality studies. The Norrkoping, ${ }^{5,6}$ Quebec $^{7,8}$ and Stockholm ${ }^{14}$ studies had unclear (or quasi) randomization, unclear allocation concealment, lacked blinded outcome assessment, and did not provide power calculation. Each also had specific additional concerns. Norrkoping ${ }^{5,6}$ did not originally include PSA screening; Quebec ${ }^{7,8}$ had very poor compliance with screening; and Stockholm ${ }^{14}$ screened patients only once and had a PSA threshold for biopsy higher than present standards. For these reasons, we do not think these trials reflect population PSA-based prostate cancer screening and we did not consider them in determining if screening is effective.

We also feel these three poor quality trials likely confuse the meta-analyses. ${ }^{1,2}$ Interestingly, despite their multiple limitations, these studies can weigh considerably in meta-analysis. For example, Norrkoping ${ }^{5,6}$ was weighted as $13 \%$ of the 2010 meta-analysis ${ }^{1}$ of prostate cancer mortality, but had only $1 \%$ of the total number of men in the screening arm (1494/143 860); and $3 \%$ of the total population (9026/294 448). While this weighting is normal in meta-analytic methods, it is important to remember the limitations of studies that account for a relatively large portion of the summary statistic.

The three remaining trials (PLCO, ${ }^{9,10}$ ERSPC $^{11}$ and Göteborg ${ }^{13}$ ) are high quality. Unfortunately, the $\mathrm{PLCO}^{9}$ suffered from poor compliance (high contamination) in the no-screening arm. At the trial outset the authors outlined how poor compliance in either arm would affect the power to identify the anticipated $20 \%$ reduction in prostate cancer mortality with screening. They had anticipated a $90 \%$ compliance with screening and $20 \%$ non-compliance with no screening, ${ }^{10}$ but got $86 \%$ and $52 \%$, respectively. ${ }^{9}$ Although we felt the PLCO was well-designed, we were concerned with how compliance may influence the interpretation of prostate screening effects. While we did not dismiss PLCO, we felt ERSPC ${ }^{11}$ and Göteborg, ${ }^{13}$ which did not have the same challenge with compliance, better reflect the effect of a PSA-based prostate cancer screening (compared to no screening).

\section{Does PSA screening decrease prostate cancer mortality?}

Based on the best available trials, ${ }^{11,13}$ we concluded that prostate cancer screening, specifically PSA, does reduce prostate cancer mortality. The number needed to screen to prevent one prostate cancer death at nine and 14 years of follow-up were 1410 and 293, from the ERSPC ${ }^{11}$ and Göteborg studies, ${ }^{13}$ respectively. The corresponding number needed to treat to prevent one prostate cancer death at nine and 14 years of follow-up were 48 and 12, from the ERSPC ${ }^{11}$ and Göteborg ${ }^{13}$ studies, respectively. In comparison, the number needed to screen with mammography to prevent one breast cancer death in women $\geq 50$ years of age is 1235 and 614 for 7 and 13 years, respectively. ${ }^{22}$ The number needed to screen with fecal occult blood testing to prevent one colorectal cancer death is 617 over 12 to 18 years. ${ }^{23}$ Prostate cancer screening, similar to the other accepted cancer screening programs, does not modify overall mortality.

\section{Other important issues}

It is not the authors' intent to advocate for prostate cancer screening. Many aspects of prostate cancer screening are 
Allan et al.

\begin{tabular}{|c|c|c|c|c|c|c|c|c|}
\hline & \multicolumn{2}{|c|}{ No. randomized } & \multicolumn{2}{|c|}{ Power determination } & \multicolumn{2}{|c|}{$\begin{array}{l}\text { Percent screened in } \\
\text { each arm* }\end{array}$} & \multicolumn{2}{|c|}{$\begin{array}{l}\text { Prostate cancer mortality } \\
\text { (rate ratio unless noted) }\end{array}$} \\
\hline & Screening & No screening & $\begin{array}{l}\text { Power } \\
\text { calculated }\end{array}$ & $\begin{array}{l}\text { Corrected for } \\
\text { compliance }\end{array}$ & $\begin{array}{l}\text { Screening } \\
\text { arm }\end{array}$ & $\begin{array}{l}\text { No screening } \\
\text { arm }\end{array}$ & Intention to screen & Per protocol \\
\hline $\begin{array}{l}\text { Norrkoping, } \\
\text { Sweden } 5,6\end{array}$ & $1,494^{\dagger}$ & 7,532 & $\mathrm{n} / \mathrm{a}$ & $\mathrm{n} / \mathrm{a}$ & $83 \%^{\S}$ & $\mathrm{n} / \mathrm{a}$ & $1.04(0.64,1.68)^{* *}$ & $\mathrm{n} / \mathrm{a}$ \\
\hline Quebec City ${ }^{7,8}$ & 31,133 & 15,353 & $\mathrm{n} / \mathrm{a}$ & Unclear & $23.6 \%$ & $7.3 \%^{+t}$ & $\begin{array}{c}1.01(0.76,1.34)^{\ddagger \ddagger} \\
(\mathrm{RR})\end{array}$ & $\begin{array}{c}0.36(0.19,0.65) \\
\text { (RR) }\end{array}$ \\
\hline PLCO (USA) $)^{9,10}$ & 38,343 & 38,350 & Yes & $\begin{array}{l}\text { Yes (under- } \\
\text { estimated) }\end{array}$ & $86 \%$ & $52 \%$ & $\begin{array}{c}1.11(0.83,1.50)^{\S \S} \\
(\mathrm{RR})\end{array}$ & $\mathrm{n} / \mathrm{a}$ \\
\hline $\begin{array}{l}\text { ERSPC } \\
\text { (Europe) })^{11,12}\end{array}$ & 72,890 & 89,353 & Yes & Yes & $82.2 \%$ & $15.4 \% * * *$ & $0.80(0.65,0.98)$ & $0.69(0.51,0.92)$ \\
\hline $\begin{array}{l}\text { Goteborg } \\
\text { (Sweden) })^{13}\end{array}$ & 9,952 & 9,952 & Yes & Yes & $76.1 \%$ & “low" & $0.56(0.39,0.82)$ & $0.44(0.28,0.68)$ \\
\hline Stockholm ${ }^{14}$ & 2,400 & 24,202 & $\mathrm{n} / \mathrm{a}$ & $n / a$ & $74 \%$ & $n / a$ & $1.10(0.83,1.46)$ & $1.04(0.75,1.45)$ \\
\hline
\end{tabular}

*Percent screened: the percent in the two groups that had some level of prostate cancer screening. ${ }^{\dagger}$ Quasi-randomized: 1 in 6 randomized to screening and remainder served as control. ${ }^{\mathrm{s}} \mathrm{From}$ the first two rounds of screening. ${ }^{* *}$ Taken from the meta-analysis ${ }^{1}$ (not provided in the 15 year follow-up publication ${ }^{5}$. ${ }^{\text {t† }}$ Authors stated the level of contamination of the unscreened group could not be assessed but do show that 7.3\% of the men in the control "no screening" group were screened. "¥Taken from the meta-analysis" (not provided in the publication with longer follow-up). ${ }^{5 s}$ At 10 years of follow-up. ***Data were available in the Rotterdam portion of ERSPC where $30.7 \%$ of the population randomized to no screening received at least one PSA. An estimated $50.2 \%$ of these were asymptomatic, suggesting those PSA tests were for screening. Therefore, an estimated $15.4 \%$ of those randomized to no screening received some screening (in one population). ${ }^{11}$ The meta-analysis quotes a contamination rate of $20 \%$ in the control group but it is not clear if this a power calculation estimate or actual results from the study. n/a: not available; RR: risk ratio; PLCO: Prostate, Lung, Colorectal and Ovarian trial; ERSPC: European Randomized Study of Screening for Prostate Cancer study.

uncertain and, even if known, would likely present significant challenges in a risk-benefit analysis for populationbased screening. Positive PSA test results of 3 to $10 \mathrm{ng} / \mathrm{mL}$ has an approximate $70 \%$ chance of being false positive and, after 3 to 4 screening tests, there is a $12 \%$ to $13 \%$ risk of a false positive test. ${ }^{24,25}$ The impact of these results are not fully understood but, up to one year after testing, more men with false positive results worry about prostate cancer than men with negative results $(26 \%$ vs. $6 \%, p<0.001) .{ }^{26}$

The workup of positive PSA tests frequently includes TRUSguided biopsy. $\mathrm{PLCO}^{9}$ reported complications rates of $0.7 \%$, while the Rotterdam study (included in ERSPC) ${ }^{27}$ reported minor complications, such as hematuria lasting more than 3 days in $23 \%$ of participants and major complications, such as fever requiring antibiotics in 3.3\% and hospitalization in $0.5 \%$. By comparison, only $0.15 \%$ of core-needle biopsies for breast cancer result in infections requiring antibiotics. ${ }^{28}$

If prostate cancer is confirmed by biopsy, treatment interventions pose more significant risks to health and quality of life. Of the 1849 men diagnosed with prostate cancer in the Göteborg trial, 829 progressed to radical prostatectomy. ${ }^{29}$ The sub-study, examining the harm associated with radical prostatectomy, found that for every 10000 men screened over 14 years, 34 prostate cancer deaths will be prevented (number needed to screen: 293) at the cost of 120 more men with impotence or sexual inactivity, and 25 more men with urinary incontinence. ${ }^{29}$ The cost-effectiveness and qualityof-life information remains unclear, but will likely further complicate the ability to make clear recommendations for broad population-based screening.

If all of the factors of risk/benefit could be weighed accordingly and large-scale population-based screening was recom- mended, resource requirements could limit or prohibit broad application. With more men referred for evaluation, biopsy and treatment, any recommendations will have to consider costs and the availability of resources, such as physicians (urologist, family physicians and oncologists) and hospitals.

\section{Conclusion}

Recent meta-analyses ${ }^{1,2}$ suggesting PSA screening does not reduce prostate cancer-specific mortality are likely unreliable due to inclusion of trials with serious limitations. In high quality studies of prostate cancer screening (particularly PSA) in which compliance is similar to that anticipated in the study design, there is good evidence that prostate cancer mortality is reduced. ${ }^{11,13}$ The numbers needed to screen are at least as good as those of mammography for breast cancer and fecal occult blood testing for colo-rectal cancer. However, the high rate of false positives (and associated worry), the risk associated with prostate biopsy and the serious consequences of prostate cancer treatment must be weighed against the advantage of reduced cancer specific mortality. It is hoped that quality-of-life information and associated cost-effectiveness evaluations will further our understanding. Oversimplification of this complex problem and the potentially confusing evidence does not serve to enhance an honest and well-informed debate on the pros and cons of prostate cancer screening.

Competing interests: This paper received no directed funding. The guideline work is funded by Toward Optimized Practice. All authors worked on the Towards Optimized Practice Guideline of Prostate Cancer, but that organization did not initiate or participate in this article. Dr Chetner has received funding for travel and presentation from GSK and for presentation from Lilly. 
The continuing debate on PCa screening

This paper has been peer-reviewed.

\section{References}

1. Diulbegovic M, Beyth RJ, Neuberger MM, et al. Screening for prostate cancer: systematic review and metaanalysis of randomised controlled trials. BMJ 2010;341:c4543. http://dx.doi.org/10.1136/bmi.c4543

2. lic $D, O^{\prime}$ Connor $D$, Green $S$, et al. Screening for prostate cancer: an update Cochrane systematic review. BJU Int 2011;107:882-91.

3. Ioannidis JP, Patsopoulos NA, Evangelou E. Uncertainty in heterogeneity estimates in meta-analyses. BMJ 2007;335:914-6.

4. Liberati A, Altman DG, Tetzlaff J, et al. The PRISMA statement for reporting systematic reviews and meta-analyses of studies that evaluate health care interventions: explanation and elaboration. PLOS Med 2009;6:e1000100. Epub $2009 \mathrm{Jul} 21$.

5. Sandblom $G$, Varenhorst $E$, Löfman 0 , et al. Clinical consequences of screening for prostate cancer: 15 years follow-up of a randomised controlled trial in Sweden. Eur Urol 2004;46:717-23.

6. Varenhorst E, Carlsson P, Capik E, et al. Repeated screening for carcinoma of the prostate by digital rectal examination in a randomly selected population. Acta Oncol 1992;31:815-21.

7. Labrie F, Candas B, Cusan L, et al. Screening decreases prostate cancer mortality: 11-year follow-up of the 1988 Quebec prospective randomized controlled trial. Prostate 2004:59:311-8.

8. Labrie F, Candas B, Dupont A, et al. Screening decreases prostate cancer death: first analysis of the 1988 Quebec prospective randomized controlled trial. Prostate 1999;38:83-91.

9. Andriole GL, Crawford ED, Grubb RL III, et al. Mortality results from a randomized prostate-cancer screening trial. N Engl J Med 2009;360:1310-9.

10. Prorok PC, Andriole GL, Bresalier RS, et al. Design of the Prostate, Lung, Colorectal and Ovarian (PLCO) Cancer Screening Trial. Control Clin Trials 2000;21 (6 Suppl):273S-309S.

11. Schroder FH, Hugosson J, Roobol MJ, et al. Screening and prostate-cancer mortality in a randomized European study. N Engl J Med 2009;360:1320-8.

12. Roobol MJ, Kerkhof $M$, Schröder FH, et al. Prostate cancer mortality reduction by prostate-specific antigenbased screening adjusted for nonattendance and contamination in the European Randomised Study of Screening for Prostate Cancer (ERSPC). Eur Urol 2009;56:584-91.

13. Hugosson J, Carlsson S, Aus G, et al. Mortality results from the Goteborg randomised population-based prostate-cancer screening trial. Lancet Oncol 2010;11:725-32.

14. Kiellman A, Akre 0, Norming U, et al. 15-year followup of a population based prostate cancer screenings study. J Urol 2009;181:1615-21.
15. Jegu J, Tretarre B, Grosclaude P, et al. Results and participation factors to the European randomized study of screening for prostate cancer (ERSPC) with prostate specific antigen: French departments of Tarn Herault. Prog Urol 2009; 19:487-98.

16. Canfield SE. ACP Journal Club. Annual screening for prostate cancer did not reduce mortality from prostate cancer. Ann Intern Med 2009; 150:JC6-4, JC6-5.

17. Canfield SE. ACP Journal Club. Periodic screening with prostate-specific antigen testing reduced mortality from prostate cancer. Ann Intern Med 2009; 150:JC6-5, JC6-4.

18. Hoffman RM. ACP Journal Club. Population-based screening using prostate-specific antigen testing reduced prostate cancer mortality. Ann Intern Med 2010;153:JC6-9.

19. Kirby A, Gebski V, Keech AC. Determining the sample size in a clinical trial. Med J Aust 2002; 177:256-7.

20. Crawford ED, Grubb R 3rd, Black A, et al. Comorbidity and Mortality Results From a Randomized Prostate Cancer Screening Trial. J Clin Oncol 2011;29:355-61.

21. Kjellman A, Akre 0, Norming U, et al. Dihydrotestosterone Levels and Survival in Screening-Detected Prostate Cancer: A 15-yr Follow-up Study. Eur Urol 2008;53:106-11.

22. Gotzsche PC, Nielsen M. Screening for breast cancer with mammography. Cochrane Database Syst Rev 2009:CD001877.

23. Hewitson $P$, Glasziou $P$, Watson $E$, et al. Cochrane systematic review of colorectal cancer screening using the fecal occult blood test (hemoccult): an update. Am I Gastroenterol 2008;103:1541-9.

24. Croswell JM, Kramer BS, Kreimer AR, et al. Cumulative incidence of false-positive results in repeated, multimodal cancer screening. Ann Fam Med 2009;7:212-22.

25. Kilpelainen TP, Tammela TL, Maattanen L, et al. False-positive screening results in the Finnish prostate cancer screening trial. Br J Cancer 2010;102:469-74.

26. Fowler FJ Jr, Barry MJ, Walker-Corkery B, et al. The impact of a suspicious prostate biopsy on patients' psychological, socio-behavioral, and medical care outcomes. J Gen Intern Med 2006;21:715-21.

27. Raaijmakers R, Kirkels WJ, Roobol MJ, et al. Complication rates and risk factors of 5802 transrectal ultrasound-guided sextant biopsies of the prostate within a population-based screening program. Urology 2002;60:826-30

28. Bruening W, Fontanarosa J, Tipton K, et al. Systematic review: comparative effectiveness of core-needle and open surgical biopsy to diagnose breast lesions. Ann Intern Med 2010;152:238-46.

29. Carlsson $S$, Aus $G$, Bergdahl $S$, et al. The excess burden of side-effects from treatment in men allocated to screening for prostate cancer. The Göteborg randomised population-based prostate cancer screening trial. Eur J Cancer 2010 Nov 17. [Epub ahead of print].

Correspondence: Dr. G. Michael Allan, Department of Family Medicine, University of Alberta, 901 College Plaza, Edmonton, AB T6G 2C8; fax: 780-472-5192; michael.allan@ualberta.ca 